\title{
Generalized Polynomial Chaos Paradigms to Model Uncertainty in Wireless Links
}

\author{
Marco Rossi, Dries Vande Ginste, Hendrik Rogier \\ Ghent University-iMinds: Dept. of Information Technology, Technologiepark Zwijnaarde 15, \\ 9000 Ghent, Belgium, hendrik.rogier@ugent.be
}

\begin{abstract}
A stochastic framework is proposed to evaluate the effect of random effects on the overall performance of wireless links. A generalized polynomial chaos expansion is leveraged to relate the uncertainties in antenna geometry, orientation and position to the figures of merit characterizing the link. The stochastic testing procedure is proposed as a more efficient alternative to stochastic collocation, for a large number of random variables. The non-intrusive statistical framework is applied to evaluate the uncertainty on the efficiency of a wireless power transfer system.
\end{abstract}

Index Terms-stochastic antenna modelling, wireless link performance, wireless power transfer.

\section{INTRODUCTION}

In the vision of the Internet of Things (IoT), considerable attention is devoted to enhancing the capabilities of the human body by leveraging an ever closer interaction with its 'smart' surroundings. For such body-centric systems, researchers have envisioned many applications, such as patient monitoring and rehabilitation, augmented reality, and localization during rescue operations. In particular, a most interesting class of wearable systems relies on textile antennas, which benefit from being light-weight, inconspicuous and flexible. Moreover, one of the pillars of the IoT consists in replacing active RFIDs and sensors, which necessarily rely on batteries for activation and operation, by passive components fed through wireless power transfer (WPT) strategies. Therefore, we can easily picture a scenario where a transmitter powers on-body sensors via textile antennas, which embed the electronics to convert the impinging electromagnetic radiation into direct current (DC) power.

In this respect, the possibility of assessing the power transfer efficiency (PTE) of a WPT system becomes paramount. In particular, two main types of random variations affect the performance of a WPT system in which textile antennas are deployed. First, the antenna's radiation impedance undergoes random variations due to production uncertainties [1]. As a result, the receiving antenna and the embedded rectifying circuit may suffer from mismatch. Second, small random misalignments and rotations of the transmitting and receiving devices may compromise the PTE of the WPT system. Therefore, a precise statistical evaluation of the influence of these two effects on the performance of the WPT system is needed.

The traditional approach to such a problem consists of performing Monte Carlo simulations. Yet, each evaluation of the textile antenna's performance and, by extension, of the complete wireless link containing these antennas, is costly in terms of CPU-time and memory requirements, since fullwave solvers are typically applied in the analysis. Moreover, the accuracy of Monte Carlo processes only converges with the inverse of the square root of the number of processed realizations [2]. Therefore, an excessive number of costly evaluations is required, resulting in an unacceptable computation time. To overcome this limitation, in Section III we propose a stochastic collocation method (SCM) based approach, leveraging on the gPC expansions [3], [4] introduced in Section II. The formalism allows assessing the impact of position uncertainties and antenna variability on the power transfer efficiency (PTE) of WPT system. More specifically, we start from given probability density functions (PDFs) according to which the antennas' design parameters vary. Next, we introduce a generalized polynomial chaos (gPC) expansion for each antenna configuration to model the corresponding variations in its radiation characteristics. Furthermore, on a higher level, a second gPC expansion, leveraging on a very efficient technique which assess the electromagnetic interaction among arbitrarily positioned radiating devices [5], [6], quantifies the impact of both antenna variability and position uncertainties on the PTE of the system. As the number of random variables describing all uncertainties in the complete wireless link may be large, a straightforward application of the SCM technique is not efficient. To speed up the computations, in Section IV we introduce Stochastic Testing to reduce the number of realizations required to construct our statistical model. In Section V, the approach is validated on a simple WPT system consisting of a transmitting horn antenna and a receiving Industrial, Scientific and Medical (ISM) textile antenna operating at $2.45 \mathrm{GHz}$.

\section{GENERALIZED POLYNOMIAL CHAOS}

In a wireless link, several parameters affecting the system performance, such as antenna positions and orientation, will be subject to random variations. Moreover, when relying on lowcost antennas as typically applied in wearable communication systems and in the Internet of Things, also the antenna geometry and materials may be subject to significant uncertainty. Therefore, we quantify the uncertainty on the wireless system performance by first constructing a generalized polynomial chaos expansion that relates random variations in the antenna 
geometries and positions to system performance indicators. This, in turn, enables us to calculate the probability density function quantifying the uncertainty in system performance, given the statistics of the random antenna variations.

Let us now cast all input random variables into a vector $\boldsymbol{x}$. At this moment, all components of the vector are assumed to be statistically independent. Hence, each random variable $x_{k}, k=1, \ldots, K$ is described by its cumulative distribution function $\mathcal{P}^{x_{k}}$ and probability density function (PDF) $d \mathcal{P}^{x_{k}}$ in the sample space $\Omega_{k}$. The joint distribution is then given by the product of all PDFs. To determine the statistics of $y$, we make use of the Wiener-Askey scheme [7] to approximate the relationship $y=f(\boldsymbol{x})$ by the following polynomial expansion of order $L$

$$
y \approx f^{P}(\boldsymbol{x})=\sum_{\boldsymbol{l}=0}^{L} y_{\boldsymbol{l}}^{\boldsymbol{x}} \boldsymbol{\phi}_{\boldsymbol{l}}^{\boldsymbol{x}}(\boldsymbol{x}) .
$$

with $\boldsymbol{l}=\left[l_{1}, \ldots, l_{K}\right]$ a multi-index and with $l_{1}+\ldots+l_{K} \leq L$. An optimal expansion is obtained when the set of expansion polynomials $\boldsymbol{\phi}_{\boldsymbol{l}}^{\boldsymbol{x}}(\boldsymbol{x})$ consists of a product of orthogonal polynomials $\phi_{l, k}\left(x_{k}\right)$. Each such polynomial forms a complete orthogonal basis in $\Omega_{k}$ with orthogonality relation

$$
\begin{aligned}
\left\langle\phi_{i, k}^{x_{k}}\left(x_{k}\right), \phi_{j, k}^{x_{k}}\left(x_{k}\right)\right\rangle & =\int_{\Omega_{k}} \phi_{i, k}^{x_{k}}\left(x_{k}\right) \phi_{j, k}^{x_{k}}\left(x_{k}\right) d \mathcal{P}^{x_{k}} \\
& =\left|\phi_{i, k}^{x_{k}}\left(x_{k}\right)\right|^{2} \delta_{i j} .
\end{aligned}
$$

For this orthogonal set of polynomials, the Cameron-Martin convergence theorem guarantees that the expansion converges exponentially to $y=f(\boldsymbol{x})$.

Two remarks are in order here. First, in case of correlated random variables, we first decorrelate them by applying the Choleski decomposition to transform them into statistically independent variables. Second, explicit expressions for several orthogonal polynomials related to well-established distributions $d \mathcal{P}^{x_{k}}\left(x_{k}\right)$ may directly by found through the Askey scheme. Alternatively, when such polynomials are unavailable, the may be constructed by applying the modified Chebyshev algorithm.

\section{Stochastic COLLOCATION METHOD}

In this paper, we focus on non-intrusive methods, meaning that we do not require to modify the modelling or measurements procedure and that we can simply build the statistical analysis framework around the analysis or measurement procedure. We may then determine the coefficients $y_{k}^{X}$ in expansion (1) through Galerkin weighting by exploiting (2), yielding

$$
\begin{aligned}
y_{\boldsymbol{l}}^{\boldsymbol{x}} & =E\left[y(\boldsymbol{x}) \boldsymbol{\phi}_{\boldsymbol{l}}^{\boldsymbol{x}}(\boldsymbol{x})\right] \\
& =\int_{\Omega_{1}, \ldots, \Omega_{L}} y(\boldsymbol{x}) \phi_{l_{1}, 1}^{x_{1}}\left(x_{1}\right) \ldots \phi_{l_{K}, K}^{x_{K}}\left(x_{K}\right) d \mathcal{P}^{x_{1}} \ldots d \mathcal{P}^{x_{K}} .
\end{aligned}
$$

The most straightforward way of evaluation this integral consists of applying a Gauss quadrature product rule, being

$$
\begin{array}{r}
y_{\boldsymbol{l}}^{\boldsymbol{x}} \approx \sum_{i_{1}=1}^{N_{1}} \ldots \sum_{i_{K}=1}^{N_{K}} w_{i_{1}} \ldots w_{i_{K}} y\left(x_{i_{1}}, \ldots, x_{i_{K}}\right) \\
\phi_{l_{1}, 1}^{x_{1}}\left(x_{i_{1}}\right) \ldots \phi_{l_{K}, K}^{x_{K}}\left(x_{i_{K}}\right),
\end{array}
$$

with the quadrature points $x_{i_{k}}$ are found as the $N_{k}$ zeros of $\phi_{N_{k}}^{x_{k}}\left(x_{k}\right)$ in $\Omega_{k}$ and $w_{i_{k}}$ the corresponding weights. Hence, (4) requires $\prod_{k=1}^{K} N_{k}$ evaluations of the function $y=f(\boldsymbol{x})$, which results in a large number of function evaluation and is therefore not efficient for a large number random variables $K$. The efficiency can be increased by reverting to Stroud cubature rules, but even this approach suffers from the curse of dimensionality.

\section{Stochastic testing}

To avoid the curse of dimensionality, [8] introduced stochastic testing to compute the coefficients $y_{l}^{\boldsymbol{x}}$ in (1). This approach is far more efficient than Gaussian quadrature product rules and also outperforms the Stroud cubature rules for large numbers of random variables. The method starts by defining the $K$-dimensional collocation point $\boldsymbol{x}_{i}=\left[x_{i_{1}}, x_{i_{2}}, \ldots, x_{i_{K}}\right]$ as a combination of the quadrature points $x_{i_{k}}$ corresponding to the random variables $x_{k}$. Initially, a set of $R=\prod_{k=1}^{K} N_{k}$ collocation points $\boldsymbol{t}_{r}=\left[x_{r_{1}}, x_{r_{2}}, \ldots, x_{r_{K}}\right]$ is constructed through the conventional $K$-dimensional tensor product Gaussian quadrature rule. However, in the remainder of the algorithm only $M \ll R$ points $\boldsymbol{x}_{i}$ are selected by the Stochastic Testing (ST) algorithm proposed in [9]. This is done by first sorting the initial set of points according to their corresponding weights $w_{r}=w_{r_{1}} \ldots w_{r_{K}}$, in decreasing order, taking the $\boldsymbol{t}_{1}$ with the largest weight as a first collocation point $\boldsymbol{x}_{1}$. Next, the following $M \times 1$ matrix $\boldsymbol{V}$ is computed as

$$
\boldsymbol{V}=\frac{\phi_{\boldsymbol{l}}^{\boldsymbol{x}}\left(\boldsymbol{x}_{1}\right)}{\left\|\boldsymbol{\phi}_{\boldsymbol{l}}^{\boldsymbol{x}}\left(\boldsymbol{x}_{1}\right)\right\|},
$$

with $\phi_{\boldsymbol{l}}^{\boldsymbol{x}}\left(\boldsymbol{x}_{1}\right)=\left[\phi_{l_{1}, 1}^{x_{1}}\left(\boldsymbol{x}_{1}\right), \ldots, \phi_{l_{K}, K}^{x_{K}}\left(\boldsymbol{x}_{1}\right)\right]^{T}$. All the other $M-1$ collocation points $\boldsymbol{t}_{r}$ are iteratively selected as new collocation points provided they satisfy the condition

$$
\frac{\left\|\boldsymbol{v}\left(\boldsymbol{t}_{r}\right)\right\|}{\left\|\phi_{l}^{\boldsymbol{x}}\left(\boldsymbol{t}_{r}\right)\right\|}>\chi
$$

where $\chi$ is a threshold value, selected to be $10^{-3}$, and $\boldsymbol{v}\left(\boldsymbol{t}_{r}\right)$ is given by

$$
\boldsymbol{v}\left(\boldsymbol{t}_{r}\right)=\phi_{\boldsymbol{l}}^{\boldsymbol{x}}\left(\boldsymbol{t}_{r}\right)-\boldsymbol{V} \boldsymbol{V}^{T} \boldsymbol{\phi}\left(\boldsymbol{t}_{r}\right) .
$$

After selecting each point, the matrix $\boldsymbol{V}$ is updated by including the normalized vector

$$
\frac{\boldsymbol{v}\left(\boldsymbol{t}_{r}\right)}{\left\|\boldsymbol{v}\left(\boldsymbol{t}_{r}\right)\right\|}
$$

as a new column. Once $M$ collocation points are available, we compute both a matrix $A$, whose elements are defined as 
$a_{i l}=\boldsymbol{\phi}_{\boldsymbol{l}}^{\boldsymbol{x}}\left(\boldsymbol{x}_{i}\right)$, and its inverse $B$. Finally, the coefficients $y_{\boldsymbol{l}}^{\boldsymbol{x}}$ in (1) are given by

$$
y_{\boldsymbol{l}}^{\boldsymbol{x}}=\sum_{i=0}^{M} b_{i l} f^{P}\left(\boldsymbol{x}_{i}\right),
$$

where $b_{i l}$ is the $i l$-th element of matrix $B$ and $f^{P}\left(\boldsymbol{x}_{i}\right)$ is the function to be approximated, evaluated in $\boldsymbol{x}_{i}$.

\section{RESUlTs}

The stochastic framework is now applied to quantify the uncertainties in the WPT setup shown in Fig. 1, where an MI-212-1.72.45 GHz horn antenna emits a power of $10 \mathrm{dBm}$ at $2.45 \mathrm{GHz}$ to a dual-polarized textile patch antenna [10] (Fig. 2), positioned at a distance $d$ from the transmitter. The receiving antenna's nominal radiation impedance $Z_{\mathrm{RX}}$ equals $49.91-1.93 i \Omega$ at both feeds, with an isolation better than 15 $\mathrm{dB}$ between them.

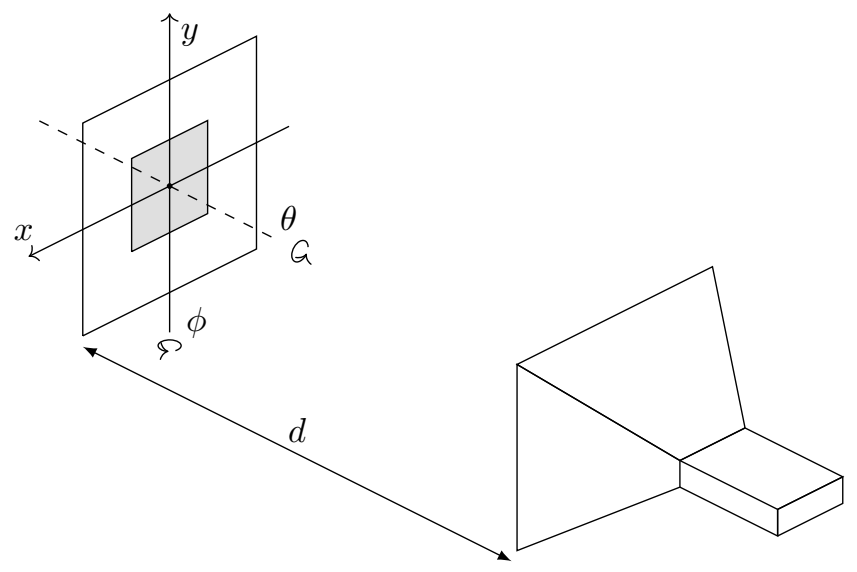

Fig. 1. WPT link under consideration.

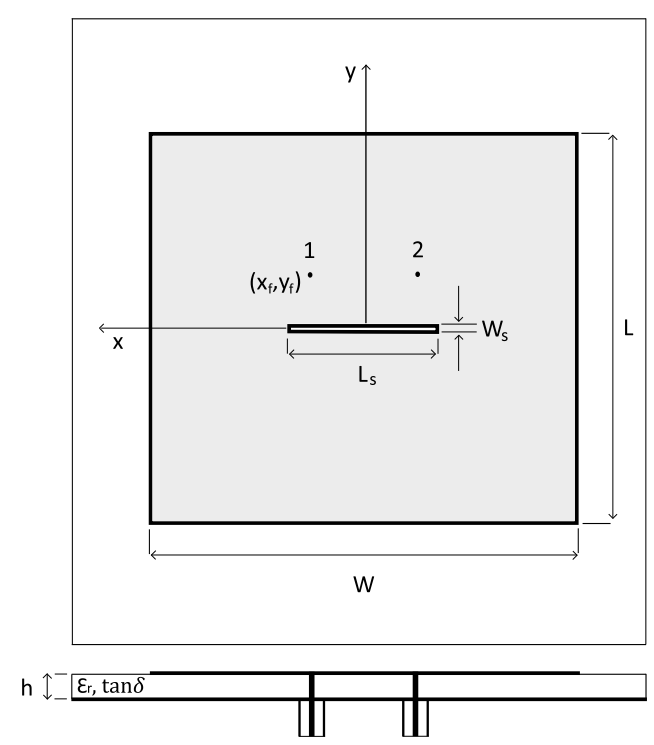

Fig. 2. Top and side view of the dual-polarized textile antenna used as a receiving antenna at $2.45 \mathrm{GHz}$. Top view. Dimensions given in Table I.
TABLE I

RECEIVING ANTENNA: NOMINAL DESIGN PARAMETERS (FIgURE 2).

\begin{tabular}{cc}
\hline Parameter & Nominal Value \\
\hline length $L$ & $44.46 \mathrm{~mm}$ \\
width $W$ & $45.32 \mathrm{~mm}$ \\
slot length $L_{s}$ & $14.88 \mathrm{~mm}$ \\
slot width $W_{s}$ & $1 \mathrm{~mm}$ \\
feed positions $\left( \pm x_{f}, y_{f}\right)$ & $( \pm 5.7,5.7) \mathrm{mm}$ \\
substrate thickness $h$ & $3.94 \mathrm{~mm}$ \\
permittivity $\epsilon_{r}$ & 1.53 \\
loss tangent $\tan \delta$ & 0.012 \\
\hline
\end{tabular}

The fast radiative near-field formalism found in [5] is leveraged to compute the wireless link efficiency $\eta_{\text {link }}$ of the WPT link, for arbitrary positions of transmitter and receiver. On the latter antenna, the rectifier together with voltage doubler and matching network (Fig. 3), provides a useful DC power. An harmonic balance simulation in ADS, yields the matching efficiency $\eta_{\text {match }}$, and the voltage doubler and rectifier efficiency $\eta_{\text {rect }}=P_{\text {inc }} / P_{\mathrm{DC}}$, with $P_{\text {inc }}=\eta_{\text {match }} \cdot P_{\mathrm{RX}}$ and $P_{\mathrm{DC}}=V_{\text {out }}^{2} / R_{L}$. Then, the overall PTE of the system is obtaned as PTE $=\eta_{\text {link }} \cdot \eta_{\text {match }} \cdot \eta_{\text {rect }}$.

\section{A. Random variables related to the antennas}

Since the transmit antenna is a standard gain horn, we only consider random variations in the receiving textile antenna. More specifically, such antenna will be subject to uncertainties in its geometrical dimensions as well as to variations in the relative permittivity $\epsilon_{r}$ of the protective foam applied as a substrate. According to [1], the most significant variations in radiation impedance $Z_{\mathrm{RX}}=Z^{r e}+j Z^{i m}$ and radiation pattern are due to random changes in the patch length $L$, the patch width $W$ and relative permittivity of the substrate. Hence, the following $\mathrm{gPC}$ expansions are put forward:

$$
\begin{aligned}
Z^{r e} & =\sum_{\boldsymbol{l}_{1}=0}^{L_{Z^{r e}}} y_{\boldsymbol{l}_{1}}^{\boldsymbol{x}} \phi_{\boldsymbol{l}_{1}}^{\boldsymbol{x}}\left(\mathbf{x}^{\mathrm{VAR}}\right) \\
Z^{i m} & =\sum_{\boldsymbol{l}_{2}=0}^{L_{Z^{i m}}} y_{\boldsymbol{l}_{2}}^{\boldsymbol{x}} \boldsymbol{\phi}_{\boldsymbol{l}_{2}}^{\boldsymbol{x}}\left(\mathbf{x}^{\mathrm{VAR}}\right) \\
A_{p q}^{r e}= & \sum_{\boldsymbol{l}_{3}=0}^{L_{p q}^{r e}} y_{\boldsymbol{l}_{3}}^{\boldsymbol{x}} \boldsymbol{\phi}_{\boldsymbol{l}_{3}}^{\boldsymbol{x}}\left(\mathbf{x}^{\mathrm{VAR}}\right) \\
A_{p q}^{i m}= & \sum_{\boldsymbol{l}_{p q}^{i m}}^{L_{p}=0} y_{\boldsymbol{l}_{4}}^{\boldsymbol{x}} \boldsymbol{\phi}_{\boldsymbol{l}_{4}}^{\boldsymbol{x}}\left(\mathbf{x}^{\mathrm{VAR}}\right) \\
B_{p q}^{r e}= & \sum_{\boldsymbol{l}_{5}=0}^{L_{B_{p}}^{r e}} y_{\boldsymbol{l}_{5}}^{\boldsymbol{x}} \boldsymbol{\phi}_{\boldsymbol{l}_{5}}^{\boldsymbol{x}}\left(\mathbf{x}^{\mathrm{VAR}}\right) \\
B_{p q}^{i m}= & \sum_{B_{p q}^{i m}}^{i m} y_{\boldsymbol{l}_{6}}^{\boldsymbol{x}} \boldsymbol{\phi}_{\boldsymbol{l}_{6}}^{\boldsymbol{x}}\left(\mathbf{x}^{\mathrm{VAR}}\right),
\end{aligned}
$$

where $\mathbf{x}^{\mathrm{VAR}}=\left[L, W, \epsilon_{r}\right] \cdot A_{p q}=A_{p q}^{r e}+j A_{p q}^{i m}$ and $B_{p q}=B_{p q}^{r e}+j B_{p q}^{i m}$ are the coefficients of the spherical 


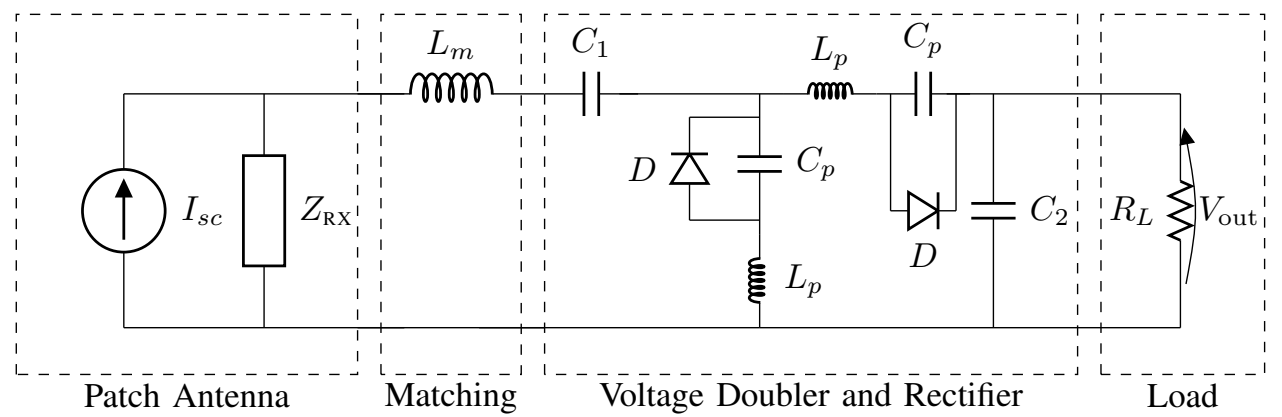

Fig. 3. The complete schematic of a rectenna element as designed and simulated in Advanced Design System (ADS).

harmonics expansion of the radiation pattern of the receiving antenna. These are leveraged for a fast evaluation of the radiative near-field link, following the formalism adopted in [5], from which the wireless link efficiency $\eta_{\text {link }}$ and the incoming power $P_{\mathrm{RX}}$, can be computed. Given the experimental data found in [1], [11], $L, W$ and $\epsilon_{r}$ are all independent Gaussian random variables (see Table II for their mean values and standard deviations). Therefore, the multivariate polynomials $\phi_{\boldsymbol{l}_{1}}^{\boldsymbol{x}}\left(\mathbf{x}^{\mathrm{VAR}}\right), \phi_{\boldsymbol{l}_{2}}^{\boldsymbol{x}}\left(\mathbf{x}^{\mathrm{VAR}}\right), \phi_{\boldsymbol{l}_{3}}^{\boldsymbol{x}}\left(\mathbf{x}^{\mathrm{VAR}}\right), \phi_{\boldsymbol{l}_{4}}^{\boldsymbol{x}}\left(\mathbf{x}^{\mathrm{VAR}}\right), \boldsymbol{\phi}_{\boldsymbol{l}_{5}}^{\boldsymbol{x}}\left(\mathbf{x}^{\mathrm{VAR}}\right), \boldsymbol{\phi}_{\boldsymbol{l}_{6}}^{\boldsymbol{x}}\left(\mathbf{x}^{\mathrm{VAR}}\right)$ in (10)-(15) correspond to products of Hermite polynomials.

TABLE II

GAUSSIAN RANDOM VARIABLES OF RECEIVE ANTENNA (FIGURE 2).

\begin{tabular}{ccc}
\hline parameter & mean value $\boldsymbol{\mu}$ & standard deviation $\boldsymbol{\sigma}$ \\
\hline$L$ & $45.3854 \mathrm{~mm}$ & $0.1268 \mathrm{~mm}$ \\
$W$ & $44.5146 \mathrm{~mm}$ & $0.1628 \mathrm{~mm}$ \\
$\epsilon_{r}$ & 1.5259 & 0.03190 \\
\hline
\end{tabular}

Although gPC expansions (10 and (11)) converge for orders $L_{Z_{\mathrm{RX}}^{r e}}=2$ and $L_{Z_{\mathrm{RX}}^{i m}}=3$, we require $L_{A_{p q}}=L_{B_{p q}}=$ 6 for the far-field expansions (12)-(15). Since one full-wave simulation of an antenna realization provides both impedance and radiation pattern data, we therefore choose the expansion order equal to 6 in all expansions (10)-(15). Then, the ST algorithm requires $M_{\mathrm{VAR}}=84$ realizations to be evaluated by the EM field simulator ADS Momentum.

\section{B. Random variables related to the link}

In nominal conditions, the WPT system (Fig. 1) operates in the radiative near-field at a distance $d=0.6 \mathrm{~m} \approx 5 \lambda$, with the antenna phase centers aligned to $x=y=0$. Furthermore, both the horn aperture and the receive patch are aligned with the $x y$-plane. In the stochastic analysis, we include small perturbation through rotations and relative translations. In the process, the transmit antenna remains stationary, concentrating all variations in the position and rotation of the receive antenna. $d, x, y, \theta$ and $\phi$ are again assumed to be independent Gaussian random variables (see Table III for more details).
TABLE III

MEAN VALUES AND STANDARD DEVIATIONS OF THE GEOMETRICAL PARAMETERS OF THE LINK (FIGURE 1).

\begin{tabular}{cccc}
\hline parameter & mean value $\boldsymbol{\mu}$ & standard deviation $\boldsymbol{\sigma}$ & $3 \boldsymbol{\sigma}$ \\
\hline$d$ & $0.6 \mathrm{~m}$ & $0.01666 \mathrm{~m}$ & $0.05 \mathrm{~m}$ \\
$x$ & $0 \mathrm{~m}$ & $0.00666 \mathrm{~m}$ & $0.02 \mathrm{~m}$ \\
$y$ & $0 \mathrm{~m}$ & $0.00666 \mathrm{~m}$ & $0.02 \mathrm{~m}$ \\
$\theta$ & $0^{\circ}$ & $10^{\circ}$ & $30^{\circ}$ \\
$\phi$ & $0^{\circ}$ & $10^{\circ}$ & $30^{\circ}$ \\
\hline
\end{tabular}

\section{Statistics of the wireless power transfer efficiency}

We now introduce a gPC expansion for the overall PTE of the WPT system:

$$
\mathrm{PTE}=\sum_{\boldsymbol{l}_{7}=0}^{L_{\mathrm{PTE}}} y_{\boldsymbol{l}_{7}}^{\boldsymbol{x}} \boldsymbol{\phi}_{\boldsymbol{l}_{7}}^{\boldsymbol{x}}\left(\mathbf{x}^{\mathrm{WPT}}\right)
$$

with $\mathbf{x}^{\mathrm{WPT}}=\left[L, W, \epsilon_{r}, d, x, y, \theta, \phi\right]$ the vector of all random variables in the link. Next, we evaluate (16) in $M_{\mathrm{PTE}}$ collocation points $\mathbf{x}_{m}^{\mathrm{WPT}}$. At each collocation point, (10)-(15) are applied to efficiently calculate both the antenna radiation impedances and their radiation patterns. After the WPT model proposed in [5] yields the position uncertainties $\mathbf{x}_{m}^{\mathrm{WPT}}$ in the WPT link, the PTE is evaluated for those collocation points. This stepwise approach limits the number of full-wave simulations required to generate the antenna macromodels. Second, these macromodels may be reused when antenna elements in the link are repositioned.

We assume the parameters $L, W, \epsilon_{r}, d, x, y, \theta$ and $\phi$ to be independent and varying according to Gaussian distributions. Therefore, the multivariate polynomials $\phi_{\boldsymbol{l}_{7}}^{\boldsymbol{x}}\left(\mathbf{x}^{\mathrm{WPT}}\right)$ in (16) consist of products of Hermite polynomials, as in Section V-A. This assumption doens't affect the generality of the analysis, since any distribution of the considered parameters can equally be dealt with. The expansion (16) converges for an order $L_{\mathrm{PTE}}=4$. Then, a number $M_{\mathrm{PTE}}=495$ of collocation points $\mathbf{x}_{m}^{\mathrm{LINK}}$, selected by means of the ST algorithm, is processed with the WPT model described in [5] and the antenna macromodels to compute the coefficients $y_{k_{7}}$ in (16).

We validate the approach by means of a Monte Carlo analysis. Thereto, we process a sample set of 10000 realizations 
of $\mathbf{x}^{\mathrm{WPT}}$, drawn according to the PDFs of the random variables in the link. The resulting curve of the cumulative distribution functions (CDF) of the PTE, as well as that based on the SCM analysis, are shown in Fig. 4. We notice that they are perfectly overlapping. In order to prove that the two CDFs correspond to the same distribution, the Kolmogorov-Smirnov test is applied. More specifically, if the maximum distance $D_{P T E}$ between the two CDFs is smaller than a threshold distance $D_{\alpha}$, the Kolmogorov-Smirnoff test accepts the null hypothesis that both the sample sets correspond to the same distribution, with a significance level $\alpha$. For $\alpha=0.05$, we find that $D_{\alpha}=0.01923$. Being $D_{P T E}=0.0085$, the hypothesis is accepted.

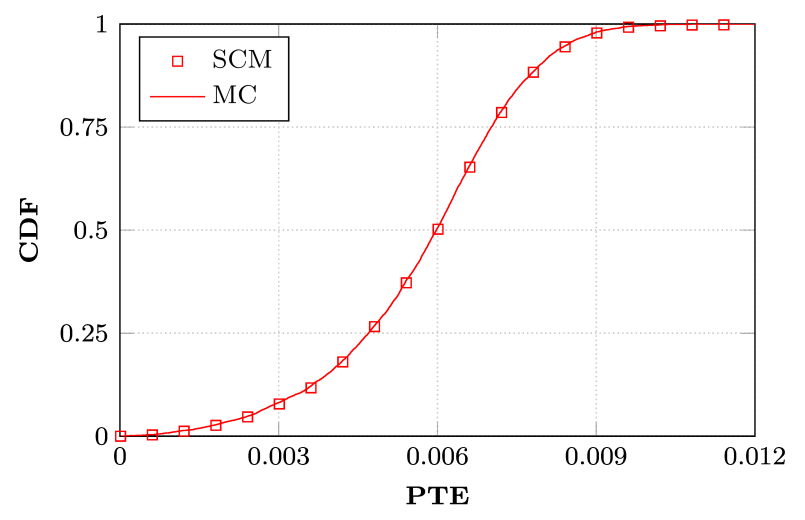

Fig. 4. Comparison between the CDFs of the power transfer efficiency (PTE) of the WPT system constructed with the advocated SCM and the MC simulations.

As a final remark, we point out that the advocated approach is more efficient and flexible than both an SCM analysis based on a single gPC expansion and a Monte Carlo analysis. More specifically, a single simulation of the $2.45 \mathrm{GHz}$ ISM band antenna in ADS Momentum requires about $15 \mathrm{~s}$. Therefore, the $M_{\mathrm{VAR}}=84$ antenna realizations $\mathbf{x}^{\mathrm{VAR}}$ are simulated in about $21 \mathrm{~min}$, whereas only $3.72 \mathrm{~s}$ are needed to construct the gPC expansions of the radiation impedance $Z_{\mathrm{RX}}$ and the coefficients $A_{p q}$ and $B_{p q}$. Then, the construction of the gPC expansion of the PTE, for which $M_{\mathrm{PTE}}=495$ collocation points $\mathbf{x}^{\mathrm{WPT}}$ are processed, requires 54 s. As a result, a complete analysis of the WPT system takes about $22 \mathrm{~min}$. Moreover, once the gPC-based macromodels (10)-(15) are available, any additional analysis of the a system using these antennas and other distributions for the position parameters takes only about $1 \mathrm{~min}$. In contrast, if a single gPC expansion is applied to directly model the overall PTE of the WPT system, 495 antenna realizations have to be processed in ADS, which requires more than $2 \mathrm{~h}$. Moreover, for any difference introduced in the distributions of the position parameters, such approach does not allow performing a quick analysis of the WPT system, as new antenna realizations need to be simulated in ADS Momentum. Finally, the validation carried out by means of the Monte Carlo method requires more than $41 \mathrm{~h}$, since 10000 realizations need to be simulated by means of ADS and the WPT model described in [5].

\section{CONCLUSION}

The efficiency of a WPT link in the radiative near-field is studied in which a textile antenna, operating in the $2.45 \mathrm{GHz}$ ISM band and connected to a rectifying circuit, is fed by a transmitting horn antenna. First, the impact of the uncertainties in the design parameters of the ISM antenna on its radiation impedance and radiation pattern is accurately modeled by means of gPC expansions. Such expansions serve as macromodels of the ISM antenna and allow calculating its radiation characteristics more efficiently than full-wave solvers. Then, an efficient model for the interaction between devices in the radiative near-field is applied and the misalignments between the antennas are also accounted for in a very precise and fast way. Finally, an SCM analysis is introduced to model the effect of both the variability of the ISM antenna and the uncertainties in its position with respect to the feeding horn antenna on the efficiency of the WPT system. The proposed approach greatly outperforms both a standard Monte Carlo analysis and an SCM analysis based on a single gPC expansion.

\section{ACKNOWLEDGMENT}

This research was partially funded by BELSPO and by the Research Foundation Flanders (FWO-Vlaanderen).

\section{REFERENCES}

[1] M. Rossi, A. Dierck, H. Rogier, and D. Vande Ginste, "A stochastic framework for the variability analysis of textile antennas," Antennas and Propagation, IEEE Transactions on, vol. 62, no. 12, pp. 6510-6514, 2014.

[2] G. Fishman, Monte Carlo: concepts, algorithms, and applications. Springer Science \& Business Media, 2013.

[3] D. Xiu and G. E. Karniadakis, "The wiener-askey polynomial chaos for stochastic differential equations," SIAM Journal on Scientific Computing, vol. 24, no. 2, pp. 619-644, 2002.

[4] D. Xiu, "Fast numerical methods for stochastic computations: a review," Communications in computational physics, vol. 5, no. 2-4, pp. 242-272, 2009.

[5] G. J. Stockman, H. Rogier, and D. V. Ginste, "Efficient modeling of interactions between radiating devices with arbitrary relative positions and orientations," IEEE Transactions on Electromagnetic Compatibility, vol. 56, no. 6, pp. 1313-1321, Dec 2014.

[6] G.-J. Stockman, D. V. Ginste, and H. Rogier, "Efficient modeling of the wireless power transfer efficiency for varying positions and orientations between transmitter and receiver," in 2015 IEEE MTT-S International Conference on Numerical Electromagnetic and Multiphysics Modeling and Optimization (NEMO), 2015, pp. 1-3.

[7] D. Xiu, "Fast numerical methods for stochastic computations: a review," Commun. Comput. Phys, vol. 5, no. 2-4, pp. 242-272, Feb. 2009.

[8] P. Manfredi, D. Vande Ginste, D. De Zutter, and F. Canavero, "Generalized decoupled polynomial chaos for nonlinear circuits with many random parameters," Microwave and Wireless Components Letters, IEEE, vol. 25, no. 8, pp. 505-507, 2015.

[9] Z. Zhang, T. El-Moselhy, I. Elfadel, and L. Daniel, "Stochastic testing method for transistor-level uncertainty quantification based on generalized polynomial chaos," Computer-Aided Design of Integrated Circuits and Systems, IEEE Transactions on, vol. 32, no. 10, pp. 1533-1545, 2013.

[10] L. Vallozzi, H. Rogier, and C. Hertleer, "Dual polarized textile patch antenna for integration into protective garments," IEEE Antennas and Wireless Propagation Letters, vol. 7, pp. 440-443, May 2008.

[11] M. Rossi, S. Agneessens, H. Rogier, and D. V. Ginste, "Stochastic analysis of the impact of substrate compression on the performance of textile antennas," IEEE Transactions on Antennas and Propagation, vol. 64, no. 6, pp. 2507-2512, June 2016. 